\title{
ORIGIN OF COSMIC RAYS
}

Edited by G. SETTI, G. SPADA, and A. W. WOLFENDALE

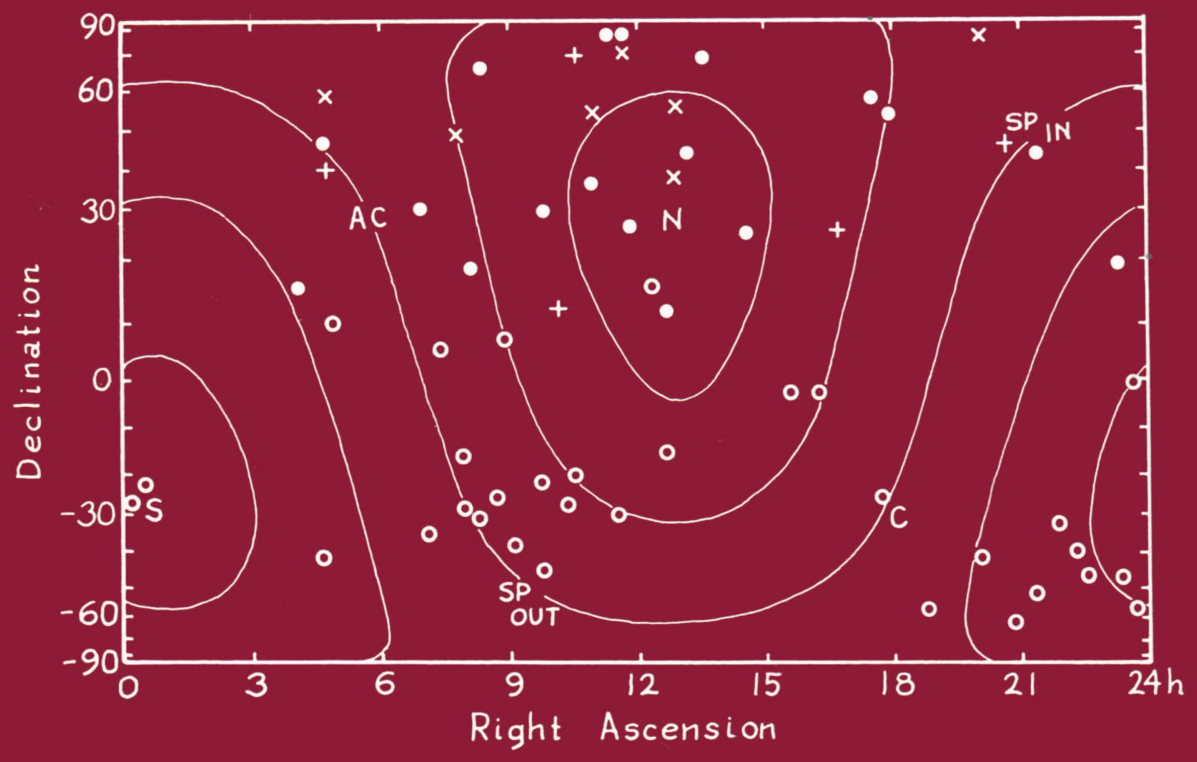


For several decades cosmic ray research has concentrated on the nuclear physical aspects of the particle beam and many important discoveries were made, notably the identification of the positron, the muon, the pion and the strange particles. More recently, however, emphasis has shifted to the astrophysical aspect both with regard to the origin of the radiation and to its relation with the other radiation fields.

These collected papers are the outcome of a symposium planned to bring together workers in all the various astronomical fields - from Radio, through Optical to Gamma Rays with Cosmic Ray physicists and to fully explore the various interrelations.

Virtually all the papers presented are reproduced here and additional short papers and resumés have also been included so that this volume represents the most up-to-date contribution available in book form. 
ORIGIN OF COSMIC RAYS 
INTERNATIONAL ASTRONOMICAL UNION

UNION ASTRONOMIQUE INTERNATIONALE

\author{
SYMPOSIUM No. 94
}

JOIŃTLY WITH INTERNATIONAL UNION! OF

PURE AND APPLIED PHYSICS

HELD IN BOLOGNA, ITALY, JUNE 11-14, 1980

\title{
ORIGIN OF COSMIC RAYS
}

\author{
EDITED BY \\ GIANCARLO SET T I \\ University of Bologna and Istituto di Radioastronomia, CNR, Bologna, Italy \\ GIANFR ANCO SPADA \\ Istituto Tecnologie e Studio Radiazioni Extraterrestri, CNR, Bologna, Italy \\ ARNOLD W. WOLFENDALE \\ Physics Department, University of Durham, U.K.
}

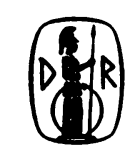

\author{
D. REIDEL PUBLISHING COMPANY \\ DORDRECHT : HOLLAND / BOSTON : U.S.A. / LONDON : ENGLAND
}

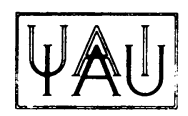


Main entry under title:

Origin of cosmic rays.

At head of title: International astronomical union; Union astronomique internationale.

1. Cosmic rays-Congresses. I. Setti, Giancarlo, 1935-

II. Spada, Gianfranco. III. Wolfendale, A. W. IV. International astronomical union.

$\begin{array}{ll}\text { QC485.8.07074 } \quad 523.01^{\prime} 97223 & 81-2574 \\ \text { ISBN 90-277-1271-9 } & \text { AACR2 } \\ \text { ISBN 90-277-1272-7 (pbk.) } & \end{array}$

ISBN 90-277-1272-7 (pbk.)

Published on behalf of

the International Astronomical Union

by

D. Reidel Publishing Company, P.O. Box 17, 3300 AA Dordrecht, Holland

All Rights Reserved

Copyright $@ 1981$ by the International Astronomical Union

Sold and distributed in the U.S.A. and Canada by Kluwer Boston Inc., 190 Old Derby Street, Hingham, MA 02043, U.S.A.

In all other countries, sold and distributed

by Kluwer Academic Publishers Group,

P.O. Box 322, 3300 AH Dordrecht, Holland

D. Reidel Publishing Company is a member of the Kluwer Group.

No part of the material protected by this copyright notice may be reproduced or utilized in any form or by any means, electronic or mechanical, including photocopying, recording or by any informational storage and retrieval system, without written permission from the publisher

Printed in The Netherlands 
P. IEYER / REVIEW OF COSMIC RAYS

H. REEVES / ISOTOPES IN GALACTIC COSIIC RAYS

W.R. Webber / The Charge and Isotopic Composition of $Z \geqslant 10$ Nuclei in the Cosmic Ray Source

C.J. Waddington, P.S. Freier, R.K. Fickle and N.R. Brewster / Isotopes of Cosmic Ray Elements from Neon to Nickel

1. Cassé, J.A. Paul and J.P. Meyer / Wolf Rayet Stars and the Origin of the ${ }^{22} \mathrm{Ne}$ Excess in Cosmic Rays

Y.V. Rao, A. Davis, M.P. Hagan and R.C. Filz / CR-39 Plastic Track I) tector Experiment for Measurement of Charge Composition of Primary Cosmic Rays

G.G.C. Palunibo and G. Cavallo / What can we learn about Cosmic Rays from the UV, Optical, Radio and X-Ray Observations of Supernova 1975c in 1100 ?

M. Giler, J. Wdowczyk and A.W. Wolfendale / Diffusion of High Energy Cosmic Rays from the Virgo Cluster

T.K. Gaisser, T. Stanev, P. Freier and C.J. Waddington / On the Detection of Heavy Primaries above $10^{14} \mathrm{eV}$

A. Ferrari and A. Masani / Interstellar and Intracluster Tunnels and Acceleration of High-Energy Cosmic Rays

J. Nishimura / Features of the High Energy Electron Spectrum

P.H. FOWLER, M.R.W. MASHEDER, R.T. MOSES, R.N.F. WALKER and A. WORLEY / ULTRA HEAVY COSMIC RAYS

W.R. Binns, R. Fickle, T.L. Garrard, M.H. Israel, J. Klarmann, E.C. Stone and C.J. Waddington / The Heavy Nuclei Experiment on HEAO-3 
R. COWSIK / PROPAGATION STUDIES RELATED TO THE ORIGIN OF COSMIC RAYS

R.J. Protheroe, J.F. Ormes and G.M. Comstock / Interpretation of Cosmic Ray Composition: the Pathlength Distribution

R.I. Epstein / Are Stellar Flares and the Galactic Cosmic Rays Related?

E.B. FOMALONT / EXTENDED RADIO SOURCES

I.I.K. PAULINY-TOTH / COMPACT RADIO SOURCES

M.J. REES / NUCLEI OF GALAXIES: THE ORIGIN OF PLASMA BEAMS

iv. Panagia and K.W. Weiler / The Common Properties of Plerions and Active Galactic Nuclei

E. Hummel / Central Radio Sources in Galaxies

L. Maraschi, R. Roasio and A. Treves / A Self--Consistent liultiple Compton Scattering Model for the $X$ and $\gamma$-Ray Emission from Active Galactic Nuclei

K.0. Thielheim / Symmetry Breaking and Invariant Mass Approach to the Spiral Structure of Calaxies

R. Silberberg and M.M. Shapiro / Neutrino Emission from Galaxies and Mechanisms for Producing Radio Lobes

M. Morini and A. Treves / $\gamma$-Ray Emission from Slow Pulsars

M.II. Shapiro and R. Silberberg / Distribution of Neutrino Fluxes

R. SANCISI and P.C. VAN DER KRUIT / DISTRIBUTION OF NON-THERMAL EMISSION IN GALAXIES

G. Sironi and G. De Amici / Cosmic Rays and Galactic Radio Noise

C.G.T. Haslam, C.J. Salter and H. Stoffel / The Al1-Sky $408 \mathrm{MHz}$ Survey

S. Kearsey, J.L. Osborne, S. Phillipps, C.G.T. Haslam, C.J. Salter and H. Stoffel / The Large-Scale Distribution of Synchrotron Emissivity in the Galaxy

R. Beck and U. Klein / Radio Emission from Nearby Galaxies at High Frequencies

W.B. BURTON and H.S. LISZT / HIGH-DENSITY, COOL REGIONS OF INTERSTELLAR IATTER IN THE GALAXY

C.J. Cesarsky and R.M. Kulsrud / Cosmic-Ray Self-Confinement in the Hot Phase of the Interstellar lledium 
M. Lachièze-Rey / Rayleigh Taylor Instabilities in the Interstellar liedium

R.J. Stoneham / Nonlinear Landau Damping of Alfven Waves and the Production and Propagation of Cosmic Rays

T.K. Gaisser, A.J. Owens and G. Steigman / Cosmic Ray Antiprotons 5-12 GeV

J. Szabelski, J. Wdowczyk and A.W. Wolfendale / Anti-Protons in the Primary Cosmic Radiation

J. TRUMPER / THE X-PAY SKY

A. Kembhavi and A.C. Fabian / Quasar Contribution to the X-Ray Background

S. Ikeuchi and A. Habe / Dynamical Behaviour of Caseous Halo in a Disk Galaxy

P. Giommi and G.F. Bignami / The Fluctuations of the Cosmic X-Ray Background as a Sensitive Tool to the Universal Source Distribution

L. SCARSI, R. BUCCHERI, G. GERARDI and B. SACCO / THE GAIMA-RAY SKY

A.W. WOLFENDALE / GAMIA RAYS FROM COSMIC RAYS

T. Montmerle, J.A. Paul and M. Cassé / Cosmic Rays from Regions of Star Formation - I. The Carina Complex

M. Cassé, T. Montmerle and J.A. Paul / Cosmic Rays from Regions of Star Formation - II. The OB Associations

J.A. Paul, M. Cassé and T. Montmerle / Cosmic Rays from Regions of Star Formation - III. The Role of T-Tauri Stars in the Rho Oph Cloud

T. Dzikowski, B. Grochalska, J. Gawin and J. Wdowczyk / High Energy $\gamma$-Rays from the Direction of the Crab Pulsar

G. Pizzichini / A New Kind of Gamma Ray Burst ?

M. Salvati, E. Massaro and N. Panagia / The Components of the Galactic $\gamma$-Ray Emission

M. Giler, J. Wdowczyk and A.W. Wolfendale / Gamma Rays from Galaxy Clusters

F. Giovannelli, S. Karakula and W. Tkaczyk / High Energy Gamma Rays from Accretion Disc

G.F. Bignami / Search for X-Ray Sources in the COS-B Gamma-Ray Error Boxes

H.J. Völk, G.E. Morfill and M. Forman / Cosmic Ray Acceleration in the Presence of Losses

C.J. Cesarsky and J.P. Bibring / Cosmic-Ray Injection into Shock-'Waves 
L. O'C. Drury and H.J. Völk / Shock Structure including Cosmic Ray Acceleration

J. Pérez-Peraza and S.S. Trivedi / Selective Effects in Cosmic Rays Induced by Coulombian Interactions with Finite

Temperature P1asmas

C. CHIUDERI / HIGH ENERGY PHENOMENA IN THE SUN

G. HAERENDEL / MAGNETOSPHERIC PROCESSES POSSIBLY RELATED TO THE ORIGIN OF COSMIC RAYS

L.G. Kocharov and G.E. Kocharov / On the Mechanism of Ceneration of Solar Cosmic Rays Enriched by Helium-3 and Heavy Elements

E. Möbius and D. Hovestadt / On ${ }^{3}$-He Rich Solar Particle Events

H.S. Ahluwalia / Cosmic Ray Evidence for the Magnetic Configuration of the Heliosphere 


\section{PREFACE}

The cosmic radiation was discovered by Hess in 1912 but its origin is still the subject of much controversy and considerable study. For several decades most workers in the cosmic ray field were interested in the Nuclear Physical aspect of the particle beam and many important discoveries were made, notably the identification of the positron, the muon, the pion and the strange particles. More recently however, emphasis has changed to the Astrophysical aspect both with regard to the origin of the radiation and to its relation with the other radiation fields.

Mindful of the increasing importance of the Astrophysical facets of the subject the Cosmic Ray Commission of IUPAP approached the High Energy Astrophysics Commission of the IAU with the suggestion of a joint Symposium on Cosmic Ray Origin. The plan was to bring together workers in all the various astronomical fields - from Radio, through optical to Gamma Rays - with Cosmic Ray physicists and to fully explore the various interrelations. The approach was received with enthusiasm and this book contains the proceedings of the ensuing Symposium, (styled IUPAP/ IAU Symposium No. 94) which was held in Bologna from 11th - 14th June 1980. Virtually all the papers presented are reproduced here.

The Scientific Organizing Committee chose the invited speakers whose papers formed the major scientific component of the meeting. Additional short contributed papers were also called for and very brief 2-page resumés (which have not been refereed) are given in the proceedings.

The Symposium was sponsored by IAU, IUPAP and the National Research Council of Italy and to these bodies the organisers express their grateful thanks. The organisers are also very indebted to the Mayor of Bologna for the warm reception offered to the participants and for permission to use the "Stabat Mater" Aula for the opening session of the Symposium. The organizers wish to express their gratitude to the secretarial staff, especially to Miss Pia Tamborrino, of the TE.S.R.E. Institute for their invaluable help offered in the organization of the meeting. 


\section{SCIENTIFIC ORGANIZING COMMITTEE}

A.W. Wolfendale (Chairman), S. Hayakawa, K.I. Kellermann, F.B. McDonald, F. Pacini, K. Pinkau, J.P. Wefel, G. Setti, I.S. Shklowsky

\section{LOCAL ORGANIZING COMMITTEE}

G. Setti (Chairman), G. Spada (Secretary),

G. Cavallo, S. Cecchini, M. Galli 


\section{LIST OF PARTICIPANTS}

Ahluwalia, H.S.', University of New Mexico, Albuquerque, U.S.A. Arons, J., University of California, Berkeley, U.S.A. Attolini, M.R., Istituto TE.S.R.E., Bologna, Italy Axford, W.I., ISI für Aeronomie, Katlenburg-Lindau, W. Germany Bartolini, C., University of Bologna, Italy Beck, R., MPI für Radioastronomie, Bonn, W. Ge rmany Belli, B.l1., Istituto Astrofisica Spaziale, Frascati, Italy Bignami, G.F., Istituto Fisica Cosmica, Milano, Italy Bijleveld, W., Sterrewacht Leiden, The Netherlands Blake, P.R., University of Nottingham, England Bloemen, J.B.G.I!., Huygens Laboratorium, Leiden, The Netherlands Bradt, H., MIT, Cambridge, U.S.A. Brini, D., University of Bologna, Italy Burton, W.B., University of Minnesota, Minneapolis, U.S.A. Camenzind, M., Universität Zürich, Switzerland Camerini, U., University of Wisconsin, Madison, U.S.A. Caraveo, P.A., Istituto Fisica Cosmica, Milano, Italy Cassé, M., CEN Saclay, Gif-sur-Yvette, France Cassiday, G., University of Utah, Salt Lake City, U.S.A. Cavaliere, A., University of Roma, Italy Cavallo, G., Istituto TE.S.R.E., Bologna, Italy Cecchini, S., Istituto TE.S.R.E., Bologna, Italy Cesarsky, C.J., CEN Saclay, Gif-sur-Yvette, France Chaliasos, E., University of Athens, Greece Chiuderi, C., University of Firenze, Italy Cini, G., Istituto Cosmo Geofisica, Torino, Italy Cowsik, R., Tata Institute of Fundamental Research, Bombay, India Dardo, M., Istituto Cosmo Geofisica, Torino, Italy Di Cocco, G., Istituto TE.S.R.E., Bologna, Italy Dilworth, C., University of Milano, Italy Drury, L., MPI für Kernphysik, Heidelberg, W. Cermany Dusi, W., Istituto TE.S.R.E., Bologna, Italy Epstein, R.I., NORDITA, Copenhagen, Denmark Ferrari, A., Istituto Cosmo Geofisica, Torino, Italy Fomalont, F.B., NRAO, Charlottesville, U.S.A. Forman, M.A., University of New York, Stony Brook, U.S.A. Fowler, P.H., University of Bristol, England Fry, W.F., University of Wisconsin, Madison, U.S.A. Frontera, F., Istituto TE.S.R.E., Bologna, Italy Gaisser, T.K., University of Delaware, Newark, U.S.A. Ga1li, M., University of Bologna, Italy Gavazzi, G., Istituto Fisica Cosmica, Milano, Italy Giler, 11., University of Lodz, Poland 
Ginzburg, V.L., Lebedev Physical Institute, Moscow, U.S.S.R. Giovannelli, F., Istituto Astrofisica Spaziale, Frascati, Italy Haerendel, G., MPI für Extraterrestrische Physik, Garching, W. Germany Haslam, G., MPI für Radioastronomie, Bonn, W. Germany Hayakawa, S., University of Nagoya, Japan Hill, P., MPI für Radioastronomie, Bonn, W. Germany Horstman, H., University of Bologna, Italy Hummel, E., University of Groningen, The Netherlands Ikeuchi, S., Hokkaido University, Sapporo, Japan Jodogne, J.C., Institut Royal Météorologique, Bruxelles, Belgium Karakula, S., University of Lodz, Poland Kellermann, K.I. NRAO, Green Bank, U.S.A. Kembhavi, A.K., University of Cambridge, England

Kiraly, P., Central Research Institute of Physics, Budapest, Hungary Koch, L., CEN Saclay, Gif-sur-Yvette, France

Kocharov, G.E., Physico-Technical Institute, Leningrad, U.S.S.R.

Kondo, I., University of Tokyo, Japan

Kota, J., Central Research Institute of Physics, Budapest, Hungary Lachièze-Rey, M., CEN Saclay, Gif-sur-Yvette, France

Lee, M.A., University of New Hampshire, Durham, U.S.A.

Linsley, J., University of New Mexico, Albuquerque, U.S.A.

Mandolesi, N., Istituto TE.S.R.E., Bologna, Italy

Maraschi, L., University of Milano, Italy

Massaro, E., University of Roma, Italy

Medina, J., Grupos Cientificos CONIE INTA., Madrid, Spain

Meyer, P., University of Chicago, U.S.A.

Michalec, A., Universytet Jagiellonski, Krakow, Poland

Möbius, E., MPI für Extraterrestrische Physik, Garching, W. Germany Montmerle, T., CEN Saclay, Gif-sur-Yvette, France

Moretti, E., Istituto TE.S.R.E., Bologna, Italy

Nash, W.F., University of Nottingham, England

Nishimura, J., University of Tokyo, Japan

Occhialini, G., University of Milano, Italy

Ormes, J.F., NASA/GSFC, Greenbelt, U.S.A.

Osborne, J.L., University of Durham, England

Pacini, F., Osservatorio Astrofisico, Firenze, Italy

Paizis, C., University of Milano, Italy

Palumbo, G., Istituto TE.S.R.E., Bologna, Italy

Panagia, N., Istituto Radioastronomia, Bologna, Italy

Paul, J., CEN Saclay, Gif-sur-Yvette, France

Pauliny-Toth, I.I.K., IMI für Radioastronomie, Bonn, W. Cermany

Pérez-Peraza, J., Universidad Nacional Autonoma de Íexico, Mexico Phillips, S., University of Durham, England

Pinkau, K., MPI für Extraterrestrische Physik, Garching, W. Germany

Pizzichini, G., Istituto TE.S.R.E., Bologna, Italy

Preuss, E., MPI für Radioastronomie, Bonn, W. Germany

Protheroe, R.J., NASA/GSFC, Greenbelt, U.S.A.

Puppi, G., University of Venezia, Italy

Ramaty, R., NASA/GSFC, Greenbelt, U.S.A.

Rao, Y.V., Emmanuel College, Lexington, U.S.A.

Rasmussen, I.L., Danish Space Research Institute, Lyngby, Denmark 
Rees, M.J., University of Cambridge, England

Reeves, H., CEN Saclay, Gif-sur--Yvette, France

Rjazhskaja, 0., Lebedev Physical Institute, Moscow, U.S.S.R.

Roasio, R., University of Milano, Italy

Rochester, G.K., Imperial College, London, England

Salvati, M1., Istituto Astrofisica Spaziale, Frascati, Italy

Sancisi, R., University of Groningen, The Netherlands

Sarkar, S., Tata Institute of Fundamental Research, Bombay, India

Scarsi, L., University of Palermo, Italy

Sequeiros, J., Grupos Cientificos CONIE INTA., Madrid, Spain

Sette, D., University of Roma, Italy

Setti, G., Istituto Radioastronomia, Bologna, Italy

Shapiro, M.M., Naval Research Laboratory, Washington, U.S.A.

Simon, G., Universität Gesamthochschule, Siegen, W. Germany

Sironi, G., Istituto Fisica Cosmica, Milano, Italy

Spada, G., Istituto TE.S.R.E., Bologna, Italy

Srinivasan, G., Raman Research Institute, Bangalore, India

Stoffel, H., MPI für Radioastronomie, Bonn, W. Germany

Stoneham, R.J., University of Cambridge, England

Thanmbyahpillai, T., Imperial College, London, England

Thielheim, K.0., University of Kiel, W. Germany

Treves, A., University of Milano, Italy

Trümper, J., IIPI für Extraterrestrische Physik, Garching, W. Germany

Trussoni, E., University of Torino, Italy

Völk, H.J., MPI für Kernphysik, Heidelberg, W. Germany

Waddington, C.J., University of Minnesota, Minneapolis, U.S.A.

Wdowczyk, J., University of Lodz, Poland

Webber, W.R., University of New Hampshire, Durham, U.S.A.

Wefel, J., University of Chicago, U.S.A.

Wilkins, D., MPI für Astrophysik, Bonn, W. Germany

Windhortst, R., Sterrewacht Leiden, The Netherlands

Wolfendale, A.W., University of Durham, England

Yodh, G.B., National Science Foundation, Washington, U.S.A.

Zaninetti, L., University of Torino, U.S.A.

Zieba, S., Universytet Jagiellonski, Krakow, Poland 